\title{
La contribución de Oscar de la Cinna a la introducción del repertorio pianístico europeo en España
}

\section{The contribution of Oscar de la Cinna to the introduction of the European piano repertoire in Spain}

\author{
Victoria Alemany \\ Conservatorio Superior de Música 'Joaquín Rodrigo' \\ victoria.alemany@csmvalencia.es \\ ORCID iD: https://orcid.org/0000-0003-1822-5817
}

\section{RESUMEN}

El público español, lo mismo que el del resto de Europa y América, prefirió la lírica -ópera, zarzuela, sainete lírico y demás obras teatrales cantadas- durante la mayor parte del siglo XIX. La música instrumental -pianística, camerística y sinfónica-, por tanto, tuvo que abrirse paso en un entorno poco propicio progresiva y lentamente generando un interesante proceso por el cual, tanto los intérpretes instrumentales como la sociedad culta, aprendieron a valorarla y a disfrutar interpretándola y/o escuchándola. Con la gira de conciertos efectuada por Franz Liszt entre 1844 y 1845 por algunas capitales de provincia de la España Peninsular se inició la introducción de repertorio pianístico específico en el país; sin embargo, hasta el momento no se conoce la importante contribución al proceso realizada por Oscar de la Cinna una década más tarde (185556). Este estudio se centra, precisamente, en investigar dicha cuestión.

Palabras clave: Oscar de la Cinna, Franz Liszt, piano, obras para piano, conciertos de piano.

\section{ABSTRACT}

The Spanish public, like the rest of Europe and America, preferred the lyrical music in most of the nineteenth century -ópera, zarzuela, lyrical sainete, and others-. The instrumental music -for piano solo, chamber music and symphonic music- had to break through in an environment that was not conducive, progressive and slowly, and it generated an process by which, both the instrumental interpreters and the educated society, learned to value it and to enjoy interpreting it and/or listening to it. The concert tour conducted by Franz Liszt between 1844 and 1845 by some provincial capitals of Peninsular Spain initiated the introduction of specific piano 


\section{VICTORIA ALEMANY}

repertoire, but the important contribution to the process made by Oscar de la Cinna a decade later (1855-56) is not yet known. This study focuses, precisely, on investigating this question.

Key words: Oscar de la Cinna, Franz Liszt, piano, works for piano, piano concerts.

Alemany, V. (2018). La contribución de Oscar de la Cinna a la introducción del repertorio pianístico europeo en España. Cuadernos de Investigación Musical, 2018, 6 (extraordinario), 293-312.

DOI : 10.18239/invesmusic.v0i0.1948

\section{INTRODUCCIÓN}

La pervivencia de patrones absolutistas en España hasta entrado el siglo XIX demoró, lamentablemente, la necesaria renovación de obsoletos paradigmas causantes del atraso político, social y económico que acuciaría al país durante todo ese siglo. La actividad musical española en la época se vio también fuertemente condicionada (Alonso, 2001; Cortizo/ Sobrino, 2001) y, consecuentemente, también se retrasó el proceso de arraigo del piano como elemento de sociabilidad burgués, factor crucial en este estudio.

Es sabido que los primeros fortepianos fueron conocidos puntualmente por la corte española hacia el segundo tercio del siglo XVIII (Kirkpatrick, 1985: 147-154), sin embargo el uso del instrumento ya perfeccionado no arraigó en España hasta medio siglo más tarde, aproximadamente ${ }^{1}$ : entre 1780 y 1830 comenzaría a ser usado y construido en Madrid, capital y residencia de la corte española, principalmente, pero también, aunque de forma incidental, en ciudades peninsulares determinadas, como Zaragoza (Ezquerro, 2002) ${ }^{2}$ y Sevilla (Luengo, 2008) ${ }^{3}$, y en algunas colonias (Ezquerro, 1998) ${ }^{4}$. La creación del Real Conservatorio de Música 'María Cristina' de Madrid en $1831^{5}$, centro pionero que

\footnotetext{
${ }^{1}$ Indicativo del impulso del uso del piano en España es la aparición de una edición española de Introduction to the Art of Playing on the Pianoforte [Londres, Clementi/Banger/Hyde/Collard \& Davis, 1801] en 1811, moderno método de aprendizaje pianístico que Muzio Clementi había comenzado a distribuir por Europa tan sólo diez años antes. En sus primeras páginas se publicitaban las características de los pianos, armonios y arpas que distribuía la empresa Clementi y Comp ${ }^{a}$ en España a principios del siglo XIX (Alemany, 1997: 339-340).

${ }^{2}$ En 1778 Antonio Enríquez presentó a la junta general de Real Sociedad Económica de Amigos del País de Zaragoza diversos artefactos salidos de su obrador, entre ellos «dos pianofortes de madera de haya, similares a los fabricados en Italia o Inglaterra, cuyo coste era de la mitad que los extranjeros» (Ezquerro, 2002: 153).

${ }^{3}$ La publicación Semanario Pintoresco Español (21.12.1845) informa que el sevillano Juan del Mármol regaló en 1783 un piano de factura suya a la reina $\mathrm{M}^{\mathrm{a}}$ Luisa de Parma (Luengo, 2008: 291).

${ }^{4}$ El monje-misionero agustino aragonés fray Diego Cera (*Graus, Huesca 1762; †Manila 1832), además de construir tres órganos de tubos durante su estancia en las islas Filipinas -entre ellos un célebre órgano de bambú todavía hoy en uso-, «fabricó pianofortes -el primero que se construyó en aquellas islas, que fue regalado a la esposa de Carlos IV, la reina María Luisa de Parma, en 1793-» (Ezquerro, 1998: 730).

5 Cabría indicar como precedente del Real Conservatorio de Música "María Cristina" (1831) otro anterior fundado por el músico español José Nonó $(* 1776 ;$ †1845) en 1816, que no poseyó financiación estatal pero sí contó con la autorización real y funcionó de manera semiprivada a lo largo de dos períodos: de 1816 a 1822, y después, entre 1829 y 1832 (Cuervo, 2012).
} 
LA CONTRIBUCIÓN DE OSCAR DE LA CINNA A LA INTRODUCCIÓN DEL REPERTORIO PIANíSTICO EUROPEO EN ESPAÑA

institucionalizó la docencia musical en España, confirma con rotundidad una generalización del uso del piano en la capital que, finalmente, iría extendiéndose progresivamente hacia el resto del país.

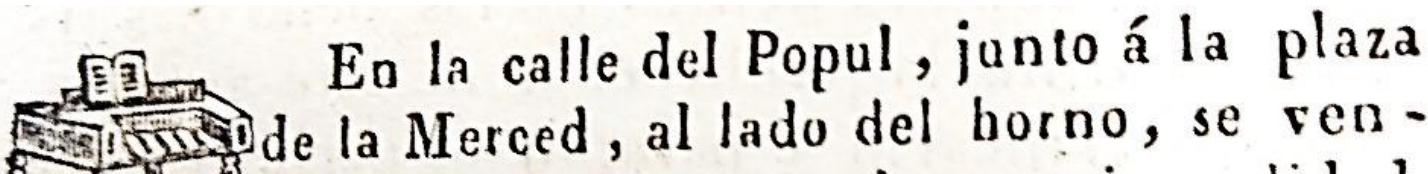 $\rightarrow-1$ den y alquilan pianos de superior calidad, á precios nódicos. Tambien se vende un mauucordio de cuatro octavas y media, el que se dará con equidad.}

Fig. 1: Imagen de un piano de mesa usada para publicitar en Valencia la venta y/o reparación de pianos.

Diario Mercantil de Valencia (12.02.1845), p. 4.

Información reciente sobre el uso del piano fuera de Madrid corrobora que condiciones sociopolíticas más favorables aplicadas después del fallecimiento de Fernando VII en España -entre 1833 y 1850 - impulsaron el proceso activando la programación de conciertos, fomentando la impartición de clases de piano y creando una incipiente industria y comercio autóctonos relacionados con la música (afinación/reparación/montaje/fabricación de pianos e impresión y venta de partituras) también en provincias.

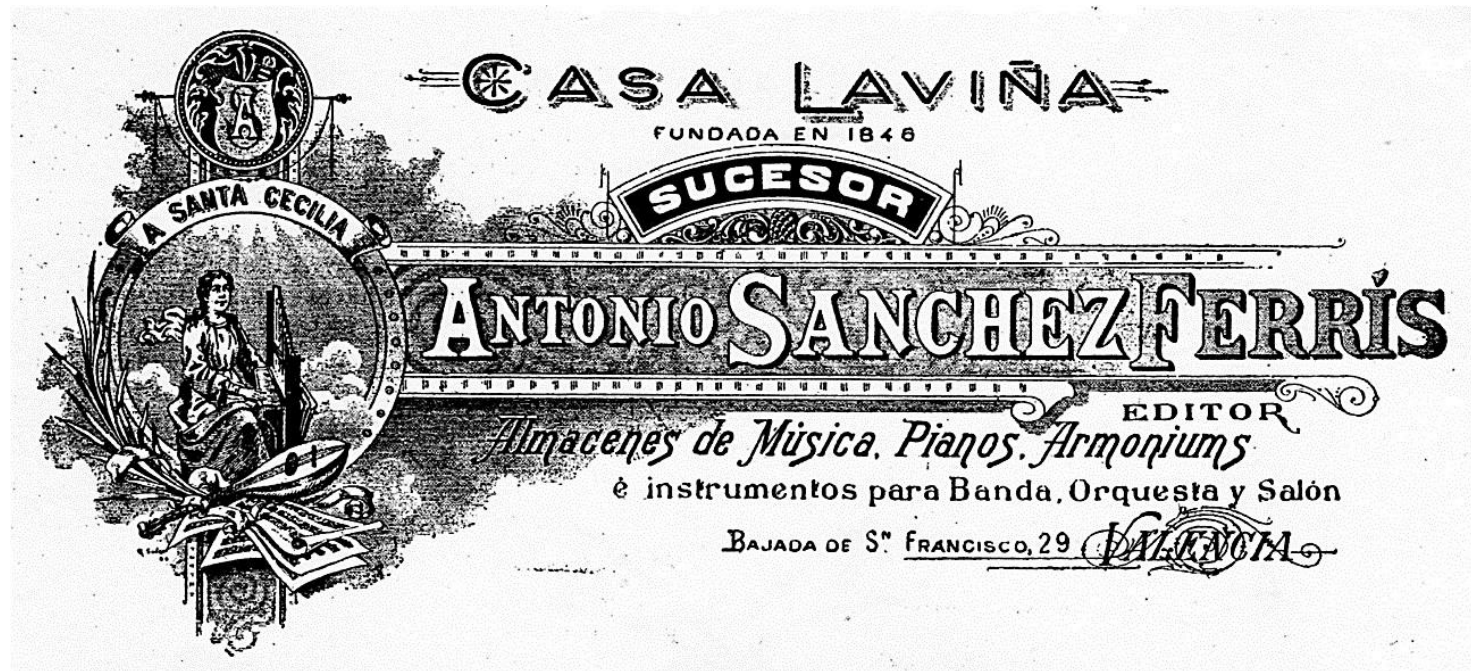

Fig. 2: Publicidad de Sanchez Ferrís donde consta que la empresa fue fundada en 1846 (Boletin Musical de Valencia, 199 [1899]) ${ }^{6}$.

\footnotetext{
${ }^{6}$ Descendiente de Casa Laviña, fue una de las dos empresas musicales autóctonas decanas de la ciudad de Valencia -la otra fue Salvador Prosper (denominada después sucesivamente Antich y Tena y Luis Tena)-.
} 


\section{Victoria ALEMANY}

Dicha documentación confirma, entre otros, los hechos siguientes:

1. La proliferación de asociaciones instructo-recreativas -denominadas Liceos-en las principales ciudades españolas entre 1830-1840 (Alonso, 2001) ${ }^{7}$, que organizaban, entre otras actividades, conciertos de forma periódica. Dichos Liceos mantenían academias donde se impartían clases de música con la finalidad de surtir su actividad musical, pues poseían orquestas integradas por sus asociados que actuaban en las habituales veladas musicales de la sociedad interpretando, casi exclusivamente, repertorio relacionado con la lírica (oberturas e intermedios orquestales, arias operísticas originales y/o transcripciones pianísticas de páginas líricas célebres). En ocasiones requerían la participación de un piano, generalmente de mesa (carré), bien para actuar como solista, bien para acompañar las actuaciones vocales [llustración 1, p. 2$]^{8}$.

2. Un significativo aumento de la oferta de docencia pianística específica -ya no de tecla antigua ambivalente para el órgano, el clave, el manucordio, etc.-, que ha podido ser documentada gracias a anuncios publicitados en fuentes hemerográficas españolas de la época 9 .

3. Aparición de talleres especializados en construcción, mantenimiento y afinación de pianos fuera de Madrid [llustración 2, p. 3].

4. Arranque de forma general en toda España de una actividad comercial musical específica dedicada exclusivamente a la difusión de partituras impresas dentro y fuera de España, y a la reparación/afinación/alquiler/venta de pianos tanto de factura nacional como extranjera [lustración 3, p. 5].

5. Giras de conciertos por España efectuadas por pianistas relevantes en la década de 1840, tanto españoles -José Miró y Anoria entre 1842 y 1843-, como extranjeros Franz Liszt entre 1844 y 1845; Émile Prudent en 1846; y Sigismund Thalberg entre 1847 y 1848-, que actualizaron en nuestro país el panorama pianístico de concierto antes de mediados del siglo XIX [llustración 4, p. 8].

\footnotetext{
7 Su aparición fue propiciada por la promulgación del Real Decreto sobre libertad de imprenta de 1834, la Constitución de 1837 y, sobre todo, por la Real Orden del 28 de febrero de 1839 que autorizaba el asociacionismo (Alonso, 2001: 22-23).

8 Precisamente después de actuar Franz Liszt en Córdoba durante la tournée que efectuó por España entre 1844 y 1845, los responsables del Liceo de dicha ciudad encargaron un piano a Louis Boisselot para utilizarlo en sus conciertos y pidieron al propio Liszt que lo eligiera personalmente en Marsella, aprovechando que pensaba pasar por allí al finalizar su gira española. El hecho se confirma en el Inventario general del Liceo de Córdoba (1842-1846), donde consta la adquisición de dos pianos en abril de 1846: uno para la cátedra de música del Liceo cordobés, que costó 2.000 reales de vellón, y otro para conciertos, cuyo precio fue de 8.186 reales de vellón (Moreno, 2004).

${ }^{9}$ Puede tomarse como prueba del exponencial incremento de la docencia pianística en una ciudad diferente a Madrid, capital y residencia de la corte española, la circunstancia de que la Guía de naturales de Valencia comenzara a publicitar a cinco profesores de piano en la ciudad en 1830, y que, sólo cuatro años después fueran ya nueve los pianistas docentes mencionados en el ejemplar correspondiente al año 1834 (Alemany, 2010: 86-87).
} 


\section{LA CONTRIBUCIÓN DE OSCAR DE LA CINNA A LA INTRODUCCIÓN DEL REPERTORIO PIANíSTICO EUROPEO EN ESPAÑA}

Una vez establecido que el proceso de arraigo del piano en España se extendió fuera de la capital entre 1830 y 1840, la investigación realizada se estructurará en tres apartados:

1. Circuito concertístico, intérpretes, tipología de los instrumentos utilizados y repertorio pianístico interpretado en España antes de 1850.

2. La gira de conciertos española de Oscar de la Cinna (1855-1856).

3. Oscar de la Cinna en la Valencia de mediados del siglo XIX.
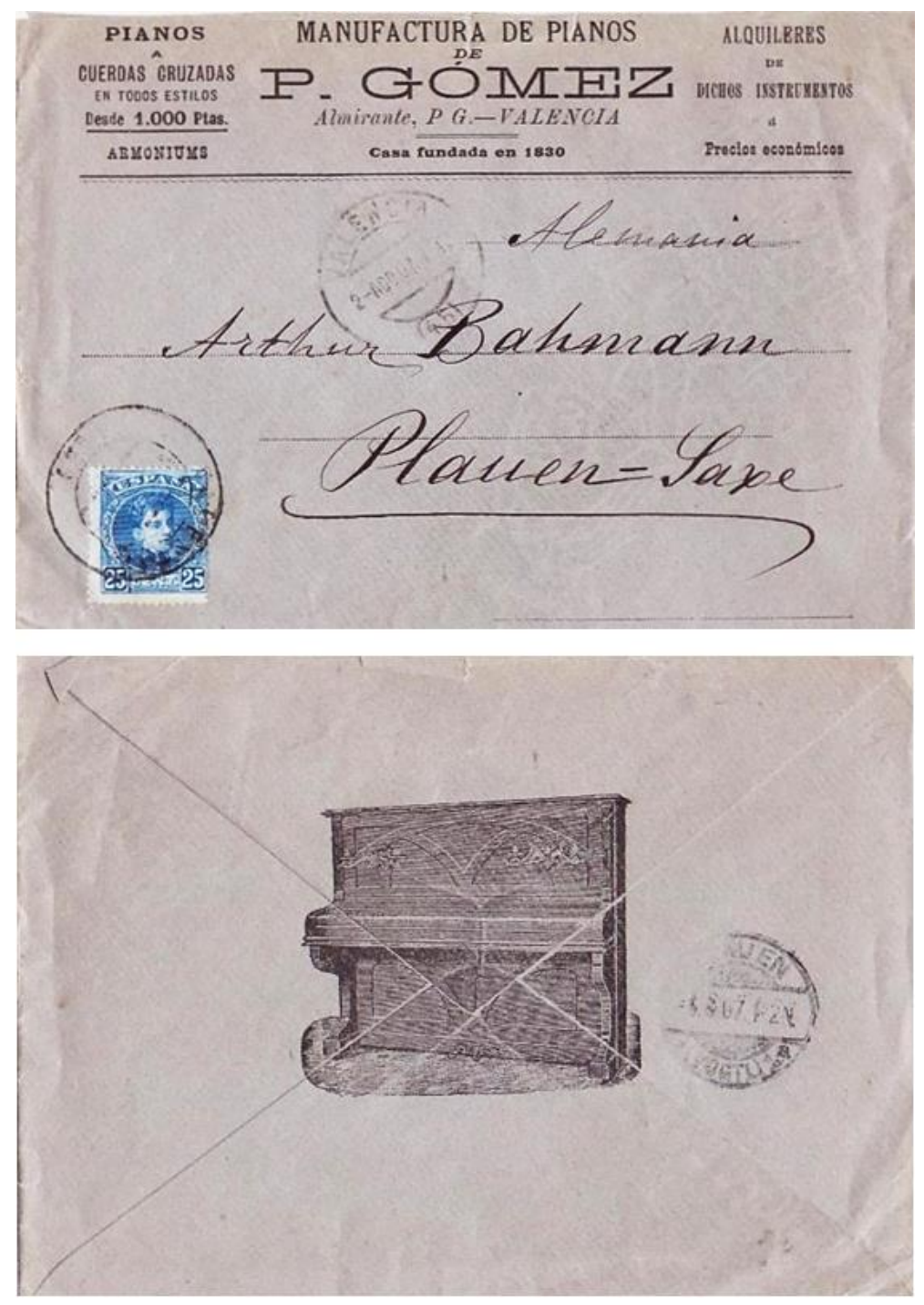

Fig. 3: Anverso y reverso del sobre usado por la empresa valenciana Pianos Gómez a principios del siglo XX10. (Archivo/Biblioteca particular de J.J. Martínez Porlán).

${ }^{10}$ En el anverso del curioso sobre que reproduzco como Ilustración 3 puede apreciarse que la fundación de los decanos talleres autóctonos data de 1830, información que confirma la que ofrecí en 2007 (Alemany, 2007: 342), en la cual daba dicho año como posible fecha de apertura del taller valenciano de Pedro Gómez 


\section{ViCTORIA ALEMANY}

\section{Circuito concertístico, pianistas, tipología DE los INSTRUMENTOS UTILIZADOS Y REPERTORIO PIANÍSTICO INTERPRETADO EN ESPAÑA ANTES DE 1850}

El recorrido efectuado por los pianistas que ofrecieron conciertos en la España Peninsular antes de 1850 muestra una evidente preferencia de los artistas por visitar la parte meridional del país ya que, aparte de la presentación obligada en la capital, sus giras comprenden únicamente dos ciudades situadas al norte, Barcelona y Zaragoza [vid. mapa en p. 8]. Dicho itinerario podría haber sido trazado buscando climas benignos para huir del frio París invernal, dado que los recorridos tuvieron lugar mayoritariamente en invierno: Franz Liszt estuvo de octubre de 1844 a abril de 1845; Émile Prudent en enero y febrero de 1846; y Sigismund Thalberg ${ }^{11}$ entre diciembre de 1847 y enero de 1848. También cabría la posibilidad de que hubiera sido aconsejado por José Miró a Franz Liszt en París, dado que ambos habrían podido trabar relación en la capital francesa entre 1829 y 1842 (de la Rosa, 2014: 141-42). Sin embargo, lo más factible es que fuera elegido en función de las capitales de provincia cuya estructura posibilitara la programación de actuaciones pianísticas con éxito, seleccionando únicamente las que contaran con salas de concierto -Teatros, Liceos o algún tipo de auditorio que pudiera albergar cierta cantidad de público- y tuvieran la posibilidad de disponer de un piano en condiciones.

La última posibilidad se confirma porque, aparte de Madrid y Barcelona, también Cádiz, Córdoba, Granada, Málaga, Sevilla, Valencia y Zaragoza disponían antes de 1850 de un Liceo -Córdoba- o de un gran Teatro -como Sevilla y Valencia-. Los pianos usados serían "de mesa" probablemente, ya que eran los que usualmente poseían los Liceos y, en casos preferentes, pianos de "de cola", bien cedidos para la ocasión por familias pertenecientes a la aristocracia o alta burguesía local ${ }^{12}$, bien propiedad de los Teatros de grandes capitales - caso de Madrid- $-^{13}$.

Antes de adentrarnos en el análisis del repertorio que fue interpretado en los conciertos conviene considerar que, como va dicho, el público español de la época prefería, en general, la lírica italiana al género sinfónico (instrumental), y este gusto musical era también el predominante en el resto de Europa. Mijail Glinka, que visitó España entre 1845 y 1847, lo constataba en una carta a su madre fechada el 8 de octubre de 1845: «Todavía no tengo

Peralta (*Teruel, 1812; †Valencia, 1887). Agradezco la aportación de la imagen a José Juan Martínez Porlán, gran amigo y estupendo profesional de la afinación de pianos.

${ }^{11}$ La España n. 2.421 [28.02.1856], p. 1; y n. 2.427 [06.03.1856] informa que Thalberg volvió a España en 1856, pero no ofreció concierto alguno al tener que marcharse precipitadamente por asuntos familiares.

12 Confirma dicha práctica lo referido en El Liceo de Córdoba n. 8 [04.12.1844], p. 4 a colación de los conciertos que iba a ofrecer Liszt en la ciudad califal «Tenemos el sentimiento de que una persona filarmónica [relevante] de esta capital haya negado su piano para tocar el Sr. Liszt. Este creemos que sea el primer caso que se haya visto en la triunfante carrera de tan célebre artista». Finalmente, parece ser que el pianista húngaro utilizó uno de los pianos Boisselot que le acompañaron durante su gira española de 1844-45.

${ }^{13}$ Los utilizados en España por Liszt entre 1844 y 1845 fueron, la mayor parte de las ocasiones, pianos de la firma Boisselot de Marsella - no puede asegurarse con seguridad si fueron uno o dos- por tanto, franceses y afines a los modernos pianos Erard con mecanismo de repetición. El propio Louis Boisselot, hijo mayor del propietario de la fábrica marsellesa, acompañó al pianista húngaro durante su tournée española para promocionar sus instrumentos [vid. nota 8, p. 4] (Alemany, 2014: 159). 


\section{LA CONTRIBUCIÓN DE OSCAR DE LA CINNA A LA INTRODUCCIÓN DEL REPERTORIO PIANíSTICO EUROPEO EN ESPAÑA}

mucha posibilidad de seguir estudiando la música nacional española, ya que en los teatros y por todas partes domina la música italiana» (Álvarez Cañibano, 1999: 90). También el pianista español Santiago de Masarnau, formado en el extranjero (Salas Villas 1999), refería por entonces en un tono pesimista quizá exagerado dado que la tendencia era general en toda Europa, la poca aceptación que poseía en España el género sinfónico y, en particular, la música que había sido compuesta para piano específicamente:

Los grandes conciertos de Mozart, Cramer, Beethoven, Hummel, Moscheles y Mendelssohn son tan conocidos de los pianistas extranjeros que suelen tocarlos todos de memoria y algunos por los doce tonos. Aquí son pocos los que saben que existen semejantes obras y de su estudio nadie se acuerda. Es verdad que para pasar por pianista en un país que se conoce tan poco y tan mal el piano no es necesario semejante estudio ni otros que son indispensables en otras partes. Para fascinar a oyentes aficionados [...] se tocan variaciones sobre un tema de ópera metiendo mucho ruido y dando porrazos al pobre instrumento [...] como si hubiera otro instrumento para el que se escribiera más música y mejor que la que se ha escrito y se escribe hoy para el piano [...] (Masarnau, 1847: 126).

No resulta extraño, por tanto, que los programas interpretados entre 1842 y 1848 por Miró, Liszt, Prudent, y Thalberg, de acuerdo con los gustos de la época, estuvieran integrados, fundamentalmente, por transcripciones operísticas y por piezas pianísticas virtuosísticas a través de las cuales cada artista trataba de sorprender al público mostrando su espectacular destreza particular de ejecución ${ }^{14}$. Merecen mención especial, asimismo, las improvisaciones al piano con las que Liszt finalizó sus actuaciones durante su gira, práctica brillantemente desarrollada por grandes compositores pianistas que le antecedieron, entre otros, Johann Sebastian Bach, Wolfgang Amadeus Mozart y Ludwig van Beethoven ${ }^{15}$. Para elaborarlas en directo, el pianista húngaro se sirvió, principalmente, de motivos musicales autóctonos que el propio público asistente le sugería ${ }^{16}$, las cuales quizá podrían haber influenciado al autor en composiciones posteriores -aunque conviene señalar que el

\footnotetext{
14 Como ejemplo puede citarse lo que Joaquín Espín y Guillén describe en un artículo dedicado a José Miró -al que califica como «primer pianista español»- con motivo de su concierto de 1842 en la Academia Filarmónica Matritense, donde interpretó una fantasía sobre Guglielmo Tell de G. Rossini -posiblemente del propio Miró-; un Nocturno de Theodor Döhler que, según indica Espín y a diferencia de los compuestos por John Field y Fréderic Chopin, constaba de cuatro partes: «un estudio para la mano izquierda; otro grande estudio para ambas manos; el trino, Capricho; y una gran fantasía de Thalberg sobre la Plegaria del Moisés [Rossini]» (Espin, 1842: 3).

${ }^{15}$ En el capítulo 14 'De l'improvisation' del Méthode des Méthodes de Piano ou Traité de l'Art de jouer cet Instrument. [París, Schlesinger, 1840] se especifica «Parmi les anciens clavecinistes, les improvisateurs les plus célèbres ont été J.S.Bach et Haendel; chez les modernes il faut placer en première ligne Mozart et Beethoven, et après eux Hummel, Moscheles et Chopin», sin mencionar a Liszt, que hacía de la improvisación una de sus especialidades.

${ }^{16}$ En Valencia, concretamente, utilizó dos melodías bailables: la denominada L'ú $i$ el dos, de carácter popular, y la Danza de los Enanos, procedente del siglo XVII, que todavía hoy puede contemplarse durante la tradicional procesión que recorre el centro histórico de la capital valenciana en la festividad religiosa de Corpus Christi (Ranch, 1945: 16-17). Además de las citadas también improvisó sobre una jota valenciana y otra aragonesa, la canción Toros del puerto de Francisco Salas, y la Tantina dels gegants catalana (Alemany, 2014: 157-158).
} 


\section{Victoria ALEMANY}

repertorio lisz̧tiano anterior a 1845 ya reflejaba un profundo interés por la música española (Gut, 1987: 225-230)-.

Como conclusión a este primer apartado cabría resaltar que, además de reminiscencias operísticas -que seguían las preferencias del gusto musical europeo decimonónico- y de las magistrales improvisaciones sobre temas autóctonos continuadoras de prácticas pianísticas dieciochescas-, los programas españoles de Liszt iniciaron la introducción en España de repertorio pianístico específico europeo, dado que incluyeron obras que no se apoyaban en la lírica: una mazurca sin determinar de Fréderic Chopin; Invitación al Vals Op. 65 [1819] y Konzerstück Op. 79 [1821] para piano y orquesta de C. M. von Weber; y tres obras propias: Grand Galop Chromatique S. 219 [1838-40], Melodías Húngaras -podría tratarse de Ungarische Nationalmelodien S 243 [1840-43]- y una Gran Marcha Húngara sin identificar -posiblemente alguna primera versión sin catalogar de alguna de sus posteriores rapsodias-.

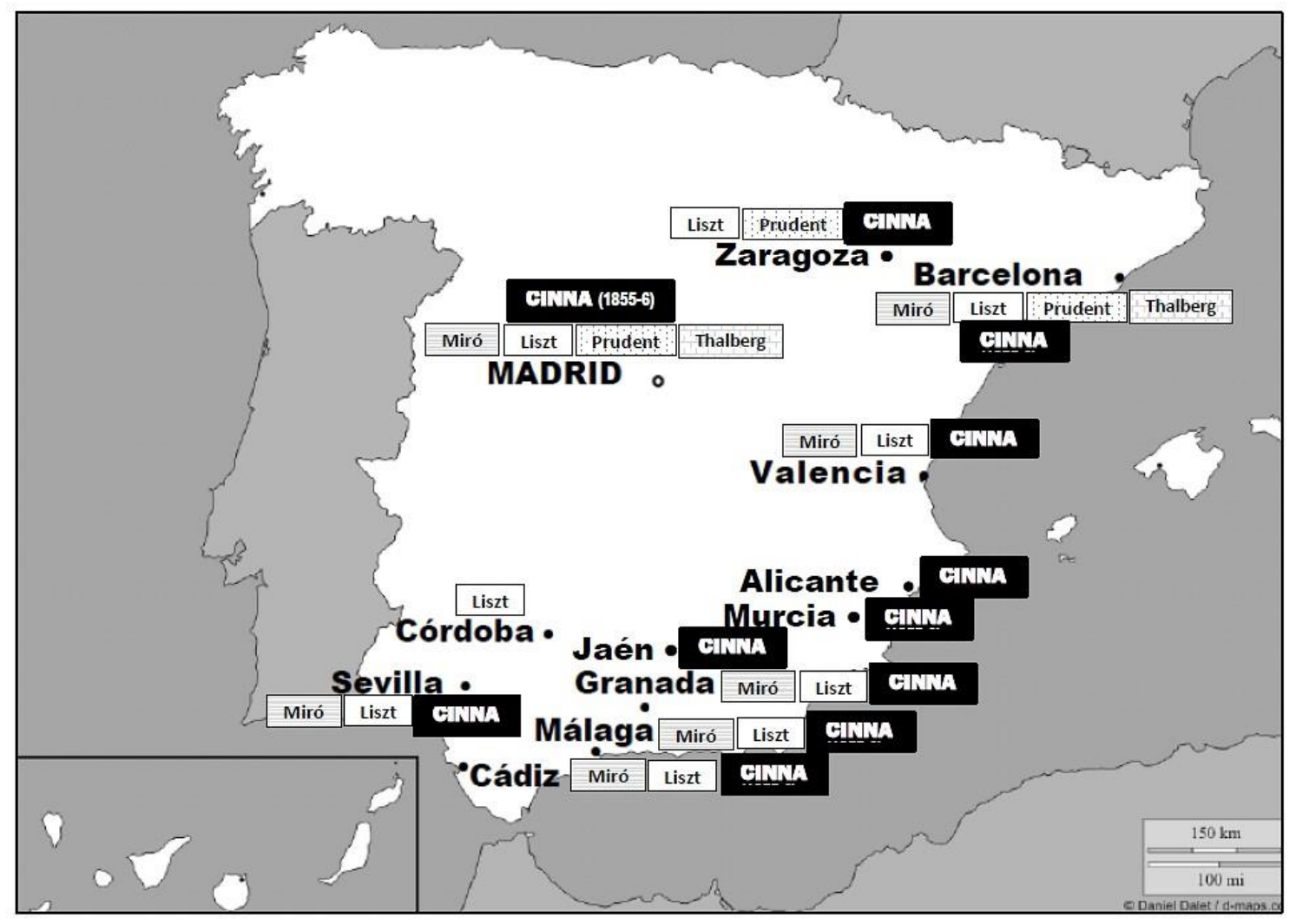

Fig. 4: Ciudades españolas visitadas por pianistas célebres antes de 1860 (Alemany, 2014) ${ }^{17}$.

José Miró y Anoria (*1815; † 1878) en 1842-1843; Franz Liszt (*1811;† 1886) en 1844-1845; Émile Prudent (*1817; † $1863)$ en 1846; Sigismund Thalberg $(* 1812 ; \nmid 1871)$ en 1847-1848;

y Oscar de la Cinna (*1836; †1906) en 1855-1856.

\footnotetext{
17 Únicamente incluye el dato de la visita de Liszt a Zaragoza un mapa con las giras del pianista húngaro que inserta Norbert Dufourcq en su Petite histoire de la Musique [París, Larousse, 1960] (Pajares, 1987: 908).
} 


\section{LA CONTRIBUCIÓN DE OSCAR DE LA CINNA A LA INTRODUCCIÓN DEL REPERTORIO PIANíSTICO EUROPEO EN ESPAÑA}

Estrenando en España modernas piezas específicamente pianísticas -ya no compuestas para clave o pianoforte indistintamente como solía suceder con repertorio anterior a 1800- Liszt contribuyó a promocionar/impulsar el género sinfónico en el país, en primer lugar; y, en segundo lugar -dato crucial para este estudio-, inició en 1844, de forma bastante temprana para España, un paulatino proceso de introducción/recepción del repertorio pianístico europeo compuesto en el siglo XIX que iría incentivándose después de 1850.

\section{LA GIRA DE CONCIERTOS ESPAÑOLA DE OSCAR DE LA CINNA (1855-1856).}

En la década de 1850, España recibió la visita de tres célebres pianistas extranjeros: Louis Moreau Gottschalk (*1829; †1869), norteamericano de ascendencia francesa y viajero incansable, que se instaló en Madrid entre 1851 y 1852; Oscar de la Cinna (*1836; †1906), húngaro, que acabaría estableciéndose, primero en Palma de Mallorca y, después, en Sevilla entre 1855 y $1856^{18}$; y Henri Herz, que visitó la capital española y algunas provincias en $1857^{19}$. Si bien la mayoría del repertorio que interpretaron versó mayoritariamente sobre transcripciones operísticas -lo mismo que el de Miró, Liszt, Prudent, y Thalberg en la década precedente-, también estrenaron piezas características compuestas a propósito de su estancia en el país como, entre otras, la espectacular obra El sitio de Zaragoza. Gran sinfonía a diez.pianos [1851] de Gottschalk (Brockett, 1992) y Los encantos de Madrid. Introducción y bolero para piano op. 189 [1857] de Herz ${ }^{20}$.

Los conciertos de Oscar de la Cinna, sin romper drásticamente con la tónica imperante y el gusto por la lírica, comenzaron a hacer valorar en España nuevos criterios musicales europeos partidarios de que el repertorio pianístico se nutriera, predominantemente, de obras compuestas para piano por autores tanto contemporáneos como de otras épocas. De hecho, el húngaro fue calificado por la prensa española como un joven pianista que había «hecho grandes estudios en el género clásico bajo la dirección de [C.] Czerny e [I.] Moscheles», y los programas que ofreció en España confirmarían rotundamente su concienzuda formación.

\footnotetext{
${ }^{18}$ Las fechas de nacimiento y muerte consignadas aparecen en el catálogo de obras del autor en la Biblioteca Nacional de Francia. Se sabe que falleció en Jerez de la Frontera, pero hay dudas sobre su nacimiento: unas fuentes señalan que vio la luz en Budapest y otras que lo hizo en Viena -capital del antiguo Imperio Austrohúngaro-. ${ }^{19}$ Lamentablemente, no he hallado datos concretos sobre las que visitó. Solo he obtenido el siguiente dato sobre su intención de desplazarse y actuar fuera de Madrid en El Museo Universal n. 9 (15.05.1857), p. 8: “El pianista Hertz nos ha dado dos funciones en el Teatro de Oriente, y ha marchado á recorrer algunas de nuestras principales ciudades, dejando recuerdos indelebles en el ánimo de los aficionados. Todos admiran sus bellas dotes musicales y la calidad de sus pianos; y no obstante los recuerdes de Liszt, de Gottschalk y otros cuyos nombres se resisten á nuestra pluma tanto como su música agradaba á nuestros oídos, ha sabido conquistarse una merecida corona de laureles".

${ }^{20}$ Dato procedente de El clamor público. Periódico Liberal (06.08.1857), p. 3.
} 


\section{VICTORIA ALEMANY}

\begin{tabular}{|c|c|c|c|c|}
\hline AUTOR & TÍTULO & LUGAR & FЕСНА & $\begin{array}{l}\text { NoDE } \\
\text { REPEIICIO } \\
\text { NES }\end{array}$ \\
\hline \multirow{2}{*}{$\begin{array}{l}\text { W. A. MOZART } \\
(* 1756 ; \dagger 1791)\end{array}$} & Concierto n. 20 KV 466 en Re Menor [1785] & $\begin{array}{l}\text { MADRID (Teatro Real) } \\
\text { VALENCIA (Salón Gómez) }\end{array}$ & $\begin{array}{l}28.05 .1855 \\
12.06 .1855\end{array}$ & 2 \\
\hline & Sonata sin determinar & $\begin{array}{l}\text { CÁDIZ (Teatro Principal) } \\
\text { SEVILLA(Teatro S. Fernando) } \\
\text { JAÉN (Casino) }\end{array}$ & $\begin{array}{l}01.12 .1855 \\
22.12 .1855\end{array}$ & 3 \\
\hline \multirow{7}{*}{$\begin{array}{l}\text { L. van BEETHOVEN } \\
\qquad(* 1770 ; \dagger 1827)\end{array}$} & Grande Sonate Patétique Op. 13 [1799] & $\begin{array}{l}\text { VALENCIA (Salón Gómez) } \\
\text { MÁLAGA (Liceo) } \\
\text { SEVILLA (?) }\end{array}$ & $\begin{array}{r}21.06 .1855 \\
11.10 .1855 \\
? .10 .1856 \\
\end{array}$ & 3 \\
\hline & Sonata Op. 26 'Marcha fúnebre’ [1800-1] & CÁDIZ (Teatro Principal) & 01.12 .1855 & 1 \\
\hline & Sonata Op. 53 'Waldstein' [1803-4] & VALENCIA (Salón Gómez) & 12.06 .1855 & 1 \\
\hline & Sonata Op. 57 'Appassionata' [1804-6] & JAÉN (Casino) & $? .02 .1856$ & 1 \\
\hline & $\begin{array}{l}\text { Concierto Op. 61a en Re Mayor [1807] } \\
\text { (transcripción del de violín Op. 61) }\end{array}$ & $\begin{array}{l}\text { MADRID (Teatro Real) } \\
\text { VALENCIA (Salón Gómez) } \\
\text { GRANADA (Teatro) } \\
\text { CÁDIZ (Teatro Principal) } \\
\text { SEVILLA(Teatro S. Fernando) }\end{array}$ & $\begin{array}{l}14.05 .1855 \\
21.06 .1855 \\
27.09 .1855 \\
01.12 .1855 \\
22.12 .1855 \\
\end{array}$ & 5 \\
\hline & $\begin{array}{c}\text { Concierto n. } 5 \text { Op. 73, en Mib Mayor [1810] } \\
\text { 'Emperador' }\end{array}$ & $\begin{array}{l}\text { MÁLAGA (Liceo) } \\
\text { SEVILLA(Teatro S. Fernando) } \\
\text { MADRID (Teatro Real) }\end{array}$ & $\begin{array}{l}17.10 .1855 \\
22.12 .1855 \\
27.031856\end{array}$ & 3 \\
\hline & Sonata Op. 81 'Les Adieux' [1809-10] & GRANADA (Teatro) & 27.09 .1855 & 1 \\
\hline $\begin{array}{l}\text { J. N. HUMMEL } \\
(* 1778 ; \dagger 1837)\end{array}$ & Concierto Op. 113 en La b Mayor [1827] & $\begin{array}{l}\text { VALENCIA (Salón Gómez) } \\
\text { ALICANTE (Ferrocarril }{ }^{1} \text { ) } \\
\text { GRANADA (Teatro) } \\
\text { SEVILLA(Teatro S. Fernando) }\end{array}$ & $\begin{array}{l}21.06 .1855 \\
02.07 .1855 \\
27.09 .1855 \\
22.12 .1855\end{array}$ & 4 \\
\hline $\begin{array}{l}\text { F. MENDELSSHON } \\
\qquad(* 1809 ; \dagger 1847)\end{array}$ & Concierto n. 1 Op. 25, en Sol Menor [1830-31] & $\begin{array}{l}\text { MÁLAGA (Liceo) } \\
\text { JAÉN (Casino) } \\
\text { MADRID (Teatro Real) }\end{array}$ & $\begin{array}{c}11 \text { y } 17.10 .1855 \\
? .02 .1856 \\
27.031856\end{array}$ & 3 \\
\hline $\begin{array}{c}\text { F. RIES } \\
(* 1784 ; \dagger 1838)\end{array}$ & $\begin{array}{l}\text { Concierto piano/orquesta sin determinar } \\
\text { [antes de 1833] }\end{array}$ & $\begin{array}{l}\text { GRANADA (Teatro) } \\
\text { MÁLAGA (Liceo) }\end{array}$ & $\begin{array}{c}27.09 .1855 \\
11 \text { y } 17.10 .1855\end{array}$ & 2 \\
\hline \multirow[b]{2}{*}{$\begin{array}{l}\text { C. M. von WEBER } \\
(* 1786 ; \dagger 1826)\end{array}$} & Sonata n. 2 Op 39 en La b Mayor [1814-16] & MÁLAGA (Liceo) & 17.10 .1855 & 1 \\
\hline & Konzerstïck Op. 79 [1821] & $\begin{array}{l}\text { MADRID (Teatro Real) } \\
\text { VALENCIA (Salón Gómez) } \\
\text { ALICANTE (Ferrocarril) } \\
\text { CÁDIZ (Teatro Principal) } \\
\text { SEVILLA(Teatro S. Fernando) } \\
\text { VALENCIA (Salón Gómez) } \\
\text { MADRID (Teatro Real) } \\
\text { SEVILLA (?) }\end{array}$ & $\begin{array}{c}14 \text { y } 28.05 .1855 \\
12.06 .1855 \\
02.07 .1855 \\
01.12 .1855 \\
22.12 .1855 \\
14.02 .1855 \\
07.04 .1856 \\
? .10 .1856\end{array}$ & 7 \\
\hline
\end{tabular}

Fig. 5: Ciudades visitadas y repertorio pianístico interpretado por Oscar de la Cinna en su gira española de 1855-1856.

El gráfico precedente recoge el repertorio y las ciudades españolas recorridas por Oscar de la Cinna entre 1855 y 1856 21 . En él puede apreciarse que el pianista húngaro visitó Madrid, Alicante, Cádiz, Granada, Jaén, Málaga, Sevilla y Valencia -aparte, documentación hemerográfica consultada informa que también tenía intención de actuar en Barcelona,

${ }^{21}$ Conviene señalar que durante la gira tuvo que detenerse en Jerez de julio a octubre de 1856 porque enfermó gravemente de viruela. Al parecer, aunque logró sanar, su rostro quedó marcado como secuela (García Tena, 1856); quizá por ello fue retratado con barba por Madrazo en 1857 [Fig. 5, p. 11]. 


\section{LA CONTRIBUCIÓN DE OSCAR DE LA CINNA A LA INTRODUCCIÓN DEL REPERTORIO PIANíSTICO EUROPEO EN ESPAÑA}

Murcia y Zaragoza ${ }^{22}$ [vid. mapa en p. 8]-, interpretando, además de obras propias ${ }^{23}$ y la Sonata n. 9 Op. 47 Kreutzer' para violín y piano de L. van Beethoven junto a intérpretes locales de determinadas ciudades que visitaba ${ }^{24}$, siete sonatas para piano solo - una sonata de Mozart sin determinar ${ }^{25}$, cinco sonatas de Ludwig van Beethoven y una de Carl Maria von Weber-; más ocho conciertos para piano y orquesta diferentes - de W. A. Mozart, L. van Beethoven, C. M. von Weber, J. N. Hummel, F. Ries ${ }^{26}$ y F. Mendelsshon (Fig. 5, p. 10)-.

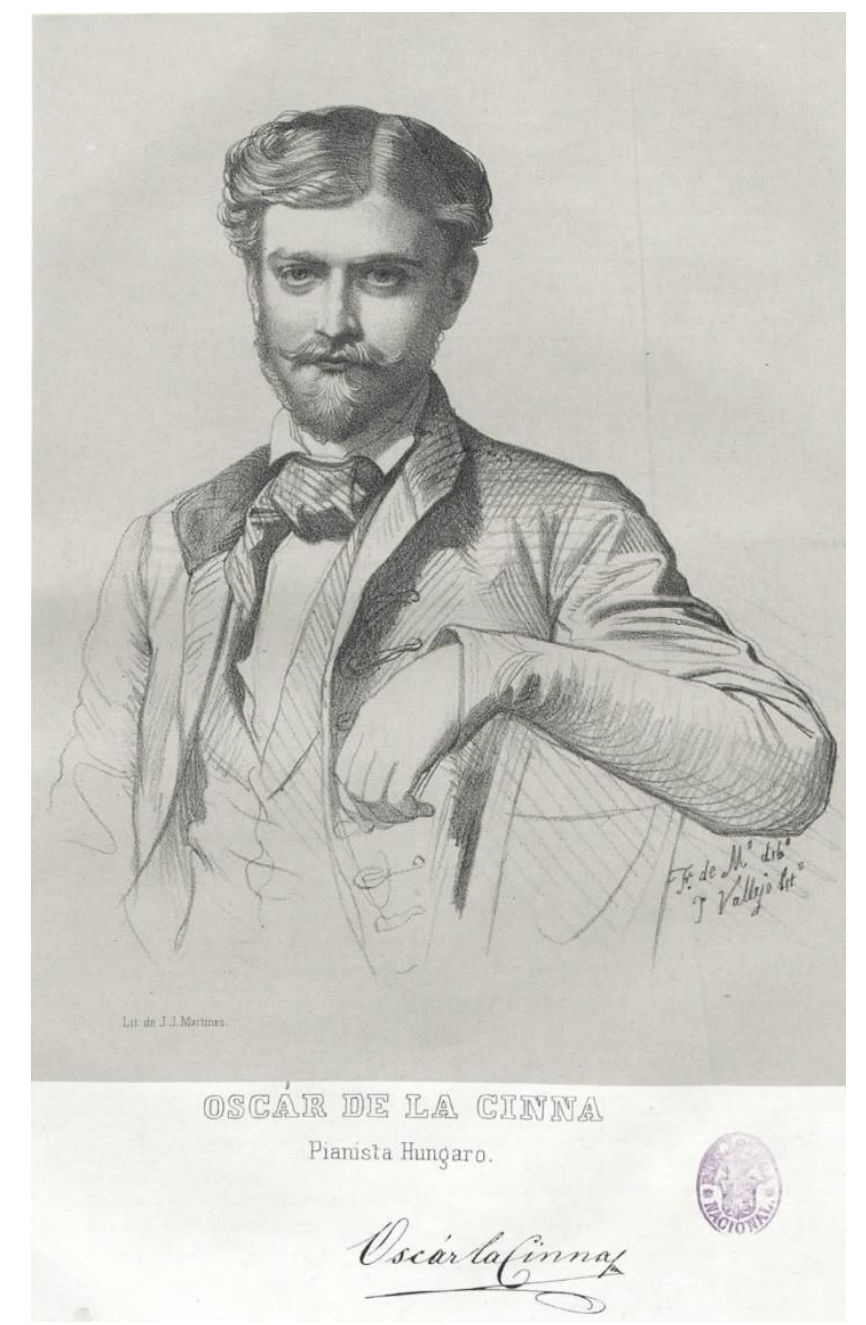

Fig. 6: Dibujo de Oscar de la Cinna realizado por Federico de Madrazo (La Zarzuela. Gaceta Musical, 70 [01.06.1857]).

\footnotetext{
22 La España n. 2332 [09.11.1855], p. 1.

${ }^{23}$ Canción caprichosa, El sueño del Cazador, una sonata (sin más datos) y unas Melodías Húngaras.

${ }^{24}$ El 14.05.1855 en el Teatro Real de Madrid junto al Sr. Pérez [Gaceta musical de Madrid 16 (20.05.1855), p. 1]; y el 01.12.1855 en el Teatro Principal de Cádiz junto a Luis Odero [Gaceta musical de Madrid 45 (09.12.1855), p. $6]$.

${ }^{25}$ La Gaceta musical de Madrid 7 (17.02.1856), p. 6 reseña que Oscar de la Cinna interpretó en su primer concierto ofrecido en el Casino de Jaén «Mozart — Andante y Presto, op. 49». Con esos confusos datos ha sido imposible identificar la pieza.

${ }^{26}$ Aunque no se especifica cuál de los nueve conciertos para piano y orquesta de Ferdinand Ries tocó en España Oscar de la Cinna, cabe indicar que todos fueron compuestos entre 1811 y 1833.
} 


\section{ViCTORIA ALEMANY}

Documentación histórica al respecto confirma que ningún pianista había interpretado en concierto público -no en salones privados- sonatas para piano originales, ni conciertos específicamente compuestos para piano y orquesta antes de que lo hiciera Oscar de la Cinna en 1855 en España, aparte de Franz Liszt ${ }^{27}$. Conviene destacar, asimismo, que las obras específicamente pianísticas interpretadas entre 1855 y 1856 por el joven pianista húngaro habían sido compuestas entre 1777 y 1828 por autores germánicos exclusivamente -W.A. Mozart; L. van Beethoven, J, N. Hummel, F. Ries, C.M. von Weber y F. Mendelssohn-, a diferencia de Franz Liszt, que había incluido en su gira de 1844-45 una mazurca de Chopin -pieza de índole popular polaca creada por un compositor autóctono-, quizá motivado por su propia inclinación hacia su nacionalismo húngaro autóctono.

Aunque la prensa musical española manifestara unánimemente aprecio incondicional por las interpretaciones de Oscar de la Cinna, al que calificó como «verdadero pianista clásico como andan pocos hoy en día por el mundo en una época en la que el piano ha salido de quicio pues se toca más para asombrar la vista que para agradar el oído», sus conciertos con programas centrados en repertorio pianístico específico -compuesto entre 1777 y 1833 c - sólo lograron alcanzar un discreto éxito de público. El texto transcrito a continuación lo confirma:

De todos los concertistas que han venido hasta el presente a la corte, solo Mr. Oscar de la Cinna se ha atrevido a ejecutar una música que, distante del gusto de la generalidad del público, requiere además el más asiduo trabajo y unas facultades privilegiadas para su empeño. Beethoven y Weber han tenido en él un verdadero intérprete, ¡Lástima y lástima muy grande que el público no comprenda cuanta abnegación se necesita en un artista que consume su talento, sus medios y su vida en el estudio de un género tan poco susceptible de popularidad, siendo al mismo tiempo la expresión más elevada del arte! (Gil, 1855: 123).

\section{OsCar de la CinNa EN la VALENCia de Mediados DEl Siglo XIX}

Tratar específicamente las dos visitas de Oscar de la Cinna a Valencia en 1855 y 1856 puede aportar valiosos datos relacionados con el asentamiento del uso del piano en el país y, más concretamente, sobre el progreso del gusto musical del denominado público 'de provincias', por lo general menos propenso a evolucionar que el de la capital y corte española, Madrid.

El número diecinueve de la Gaceta de Madrid -correspondiente al 10.06.1855informaba que el pianista húngaro salía para Valencia después de haber cosechado cierto éxito en sus dos conciertos celebrados en el Teatro Real; resulta destacable que, curiosamente, fuera la capital del Turia la primera ciudad española en la que el artista actuó fuera de la corte.

\footnotetext{
${ }^{27}$ Que, como va dicho, además de repertorio propio, tocó una mazurca sin determinar de Fréderic Chopin, y la Invitación al V als Op. 65 y el Konžertstück op. 79 de Carl Maria von Weber en Madrid y determinadas capitales de provincia españolas durante la gira que efectuó por la península entre 1845 y 1846 [vid. p. 8].
} 


\section{LA CONTRIBUCIÓN DE OSCAR DE LA CINNA A LA INTRODUCCIÓN DEL REPERTORIO PIANíSTICO EUROPEO EN ESPAÑA}

Los tres exitosos conciertos que había ofrecido el archifamoso Franz Liszt una década antes en Valencia habían tenido lugar en el Teatro Principal, sin embargo, el joven y todavía desconocido Oscar de la Cinna, que contaba tan solo diez y nueve años en 1855, no consiguió programar allí los suyos. Se presentó por primera vez ante el público levantino el martes doce de junio a las nueve de la noche en el salón que el constructor valenciano Pedro Gómez (*Teruel, 1812; †Valencia, 1887) poseía junto a las dependencias de su taller en la Plaza de San Esteban ${ }^{28}$-sede actual del Conservatorio Profesional de Música de Valencia-.

En aquel primer concierto, que fue anunciado por la prensa nacional con el apelativo de 'clásico ${ }^{29}$-posiblemente para diferenciarlo de las 'funciones filarmónicas', más comunes (Alemany, 2010: 50-52), en las cuales se interpretaba, en general, música más enraizada con la lírica (fundamentalmente oberturas, arias y dúos cantados, y transcripciones operísticas al piano) - se alternaron, como solía ser habitual, actuaciones del pianista y de la orquesta en solitario con otras que requerían la intervención conjunta de ambos. Inició la orquesta ${ }^{30}$ con la obertura de la ópera Die Zauberflöte KV 620 [1791] de Wolfgang Amadeus Mozart quizá programada para tratar de congraciarse con el público, más acostumbrado al género lírico, sin tener que alejarse del Clasicismo Vienés, en el que se centraba el concierto-; y a continuación, la orquesta acompañó a De la Cinna su interpretación del Konzerstück Op. 79 [1821] de C.M. von Weber -ya escuchado por los aficionados valencianos de manos de Liszt diez años antes-. Puso fin a la primera parte la Sonata Op. 53 'Waldstein' [1803-4] de L. van Beethoven, importante composición pianística que plantea grandes dificultades técnicas e interpretativas, con cuya ejecución el joven pianista húngaro deslumbró a los asistentes.

El programa histórico usado como fuente de documentación de este trabajo recoge que la orquesta abrió la segunda parte interpretando «Minueto y trio de la gran sinfonía en dó [sic] menor» de Beethoven, sin embargo, en los movimientos que integran su conocida Sinfonía n. 5 Op. 67 -I. Allegro con brio; II. Andante con moto; III Scherzo. Allegro; y IV. Allegro- no figura ningún minueto, por lo que no puede confirmarse que fuera ejecutada dicha sinfonía ${ }^{31}$. Seguidamente aparece un «Gran concierto de Mozart (núm. 9), Allegro y Romanza, con acompañamiento y orquesta», cuyos movimientos no se corresponden con los del Concierto n. 9 en Mi Bemol Mayor 'Jeunnehomme' KV 271 [1777] de dicho autor, con lo cual podría tratarse, en realidad, del $n$. 20, KV 466 en Re Menor [1785] cuyo segundo movimiento es una Romanza y fue interpretado de forma recurrente por De la Cinna en aquella gira española. El concierto finalizó con la interpretación de unas Melodías Húngaras a solo compuestas por el propio pianista.

\footnotetext{
${ }^{28}$ En ese mismo salón actuaría Isaac Albéniz en 1882 durante su estancia en Valencia (Alemany, 2011).

${ }^{29}$ La documentación sobre este primer concierto procede de la Gaceta de Madrid 20 [17.06.1855], p. 6.

${ }^{30}$ No se conoce si era la orquesta habitual del Teatro Principal o una academia contratada para la ocasión.

31 Podría tratarse de la Sinfonía n. 1 Op. 21 en Do Mayor [1795], pues es la a única de las nueve sinfonías de Beethoven que cuenta con minueto - a partir de la segunda este tipo de movimiento intermedio es sustituido por el scherzo-.
} 


\section{Victoria ALEMANY}

Nueve días después, a las nueve de la noche -21.06.1855, jueves-, De la Cinna volvió a actuar en el Salón Gómez de nuevo acompañado por la orquesta. En el programa figuraban, por orden, primero la obertura de la ópera Guillermo Tell [1824-29] de G. Rossini; a continuación, el Concierto Op. 61a [1807] de L. van Beethoven, transcripción para piano del propio autor del original para violín y orquesta, compuesto en 1806; y, para terminar la primera sección del concierto, la Grande Sonate Pathétique Op. 13 [1799] del compositor de Bonn.

La segunda parte se inició con la obertura de la ópera bufa en un acto Gille Ravisseur [1848] del compositor belga Albert Grisar, y a esta le siguió el Concierto Op. 113 en La bemol Mayor [1827] de J. N. Hummel. Dieron fin a la velada musical dos piezas compuestas por el intérprete húngaro: Canción caprichosa y El sueño del Cazador.

Las reseñas de dichos conciertos ${ }^{32}$ ensalzan la brillantez, expresividad y corrección de Oscar de la Cinna en la interpretación de obras cumbre del repertorio pianístico de todos los tiempos -también consideradas así en la actualidad- como son la Sonata Patética, la Sonata Waldstein y una de las últimas composiciones de Beethoven para piano y orquesta: su versión pianística del Concierto Op. 61 -sólo el Concierto n 5 Op. 73 Emperador' y la Fantasia Coral en Do Menor Op.80, escrita para orquesta y coro con piano solista, son posteriores-. Del concierto de Beethoven se ensalza expresamente «la admirable exactitud con que combinaba el ritmo ternario de la mano izquierda con el cuaternario de la derecha en pasajes prolongados ${ }^{33}$, y de la interpretación del Concierto Op. 113 de J. N. Hummel -injustamente ausente actualmente de las salas de concierto- que concluye con un Rondo à la spagniola tercer movimiento- destaca que «expresó tan perfectamente la gentileza de nuestros bailes nacionales [...] [ejecutando un] motivo alternado de dificilísimos y brillantes pasajes [que] le valió al eminente artista una nutrida salva de unánimes y repetidos aplausos».

Sintetizando, gracias a las actuaciones de Oscar de la Cinna en el hoy prácticamente desconocido Salón Gómez, propiedad del decano taller local de construcción de pianos -y probablemente usando instrumentos de factura autóctona- el público valenciano conoció en 1845 obras específicamente pianísticas de inusual interpretación en España a mediados del siglo XIX compuestas entre 1777 y 1827 -el concierto de Hummel, la más moderna, fue recibida en Valencia solo con un retraso de veintiocho años (!)-.

Cabe destacar, además, que, no citándose ningún director de orquesta, posiblemente fuera el propio pianista quien llevara también la batuta en dichos conciertos; por tanto, debió ser mérito suyo, asimismo, la interpretación por parte de la orquesta de la obertura de Gille Ravisseur [1848] de Albert Grisar que, sorprendentemente, había sido estrenada en París apenas siete años antes -el 28.02.1848-.

\footnotetext{
32 Gaceta musical de Madrid ns. 21(24.06.1855), pp. 6-7; y 22 (01.07.1855), pp. 6-7.

33 Presumiblemente el comentario se refiere a los cc. 45-50; y 71-77 del segundo movimiento Larghetto.
} 


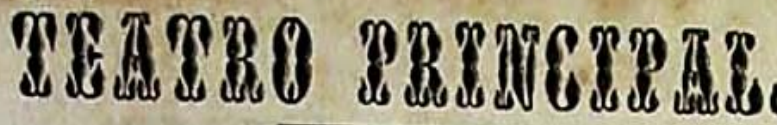

Primera funcion de abono de la segunda temporada para el juéves 14 de febrero de 1856.

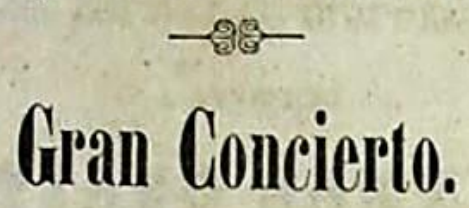

llaljándose de tránsito en esta copital el célebre piatista hủngaro

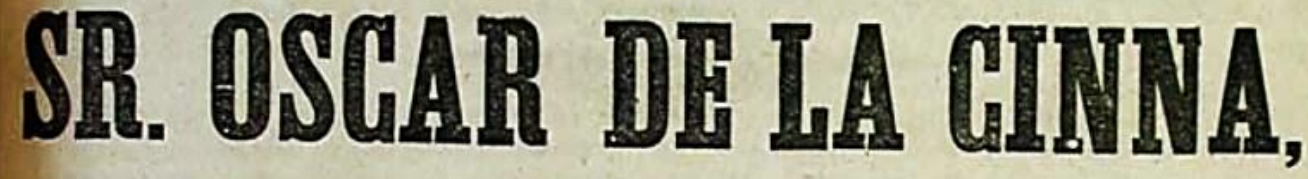

esa notabilidad clasiea que ha sido la admiracion de todos los teatros estranjeros, y cuyos recientes triunfos en el Teatro Real de Madrid, han ocupado toda la prensa de la Peninsula; ta Empresa, animada siempre de les deseos de proporcionar al público cuantas novedades estén a su alconce, ba interesado á diebo artista para dor uns representacion en este dis, por el órden siguiente:

1 Sinfonia.

2 La comedia en un acto, arreglada á la escena española, titulada

\section{POR NO ESPLICARSE...}

Por la Sra. Samaniego y los Sres. Pastrana, Torromé, Medel y Argüelles.

PRIMERA PARTE DEL CONCIERTO.

3 Sinfonia por la orquesta.

4 LARGHetTo, MARCIA y PRESTo, con acompañomiento de orquesta.

5 La comedia en un acto, arreglada á la escena española por D. Ramon do Navarrete, cuyo titulo es

\section{JA PXIXA DET TATIOH.}

Ea la que toman parte los primeros octores DOÑ JOSEFA PALMA y DON ANTONIO PIZARRoso.

6 Concierto (2' Parte.)

1 Orertura de la ópera LE NOZZE DI FIGARO.

2 ALLEGRO, ANDANTE y FINALE VIVACE, con acompañamiento de orquesta.

3 Canciones nacionales hủngaras. Piano solo.

\section{A LAS SIETE DE LA NOCHE.}

Las puertas del REAL y de CUARTE se abrirín á los que de fuera vengan á ver la funcion, segun órdea de S. E. el Sr. Capitan Geacral.

Los seãores que gusten adquirir localidades con anticipacioa, pasarán ả contadaria Ĺ las horas de costumbro.

Valencia, Impr, de J. Ferrer de Orga, á espaldas del teatro.

Fig. 7: Cartel del concierto ofrecido en Valencia por Oscar de la Cinna el 14.02.1856

(Biblioteca Histórica de la Universidad de Valencia). 


\section{Victoria ALEMANY}

Oscar de la Cinna volvería a Valencia en febrero de 1856, cuando ya estaba finalizado su primera y exitosa gira por la península y era ampliamente conocido por los aficionados españoles al piano. El recuerdo de sus valoradas actuaciones en Valencia un año antes debió propiciar, probablemente, que su posterior concierto en la capital levantina ya se efectuara en el Teatro Principal, primera sala de conciertos y representaciones teatrales de la ciudad. Sin embargo, el programa previsto no ofrecía novedades respecto a los dos ofrecidos en 1855 en el Salón Gómez, dado que volvió a interpretar el Konzerstück Op. 79 [1821] de C. M. von Weber -anunciado poco rigurosamente como Larguetto, Marcia y Presto sin citar al autor-, que ya habían tocado antes Liszt (1845) y el propio Cinna (1855); un Allegro, Andante y Finale Vivace sin identificar, con acompañamiento de orquesta, y unas Canciones nacionales húngaras, probablemente repetición de sus Melodías Húngaras para piano solo escuchadas el año anterior.

Durante esta segunda estancia también aprovecharía para participar en un concierto vocal e instrumental organizado por el Liceo valenciano que contó con la participación de la sección de música y la orquesta de aficionados de la sociedad. Precisamente gracias al anuncio de dicha actuación, posterior a su concierto del principal, he podido conocer que Oscar de la Cinna se proponía marchar «à Barcelona a proporcionarse un piano francés, propio para teatro [de gran cola (?)], á fin de dar á su vuelta algunos conciertos en el Teatro Principal» ${ }^{34}$, lo cual podría indicar que el instrumento usado por él en dicho teatro en 1856 no fuese propiedad del mismo -quizá pudiera tratarse de un piano alquilado o cedido a tal efecto-, o que no le satisficiera la calidad del utilizado. No he podido confirmar que el intérprete húngaro cumpliese su propósito, dado que no he hallado información económica alguna al respecto, y tampoco figura ningún concierto suyo con un piano nuevo entre la documentación histórica conservada actualmente sobre el Teatro Principal.

Lo expuesto demuestra que, si bien actualmente es ampliamente conocida y valorada la decisiva influencia que tuvo la gira española de Franz Liszt entre 1844 y 1845 en el proceso de arraigo del uso del piano en el país, hasta el momento no se tenía noticia de la crucial participación de Oscar de la Cinna. Entre 1855 y 1856 el pianista húngaro introdujo en nuestro país obras pertenecientes al repertorio pianístico específico germánico clásico de W. A. Mozart y L. van Beethoven- y romántico -principalmente de J.N. Hummel y F. Mendelssohn- compuestas entre 1777 y 1827, instaurando en España una práctica concertística que comenzaba por entonces a imponerse simultáneamente en el extranjero. Además, quizá por haber visitado la Península Ibérica diez años después que Liszt, De la Cinna amplió considerablemente el circuito concertístico recorrido por el primero en 1845, contribuyendo, por tanto, a ampliar el gusto musical -especialmente, pianístico- del público también en ciudades más pequeñas como Jaén y Murcia.

El panorama musical español, por tanto, que había estado casi exclusivamente centrado en la estética lírica de corte italianizante como en el resto de Europa, comenzó a renovarse y ampliarse por primera vez en 1844-45, gracias a la visita de Franz Liszt, que sorprendió al

34 Diario Mercantil de Valencia (17.02.1856), p. 1. 


\section{LA CONTRIBUCIÓN DE OSCAR DE LA CINNA A LA INTRODUCCIÓN DEL REPERTORIO PIANíSTICO EUROPEO EN ESPAÑA}

público nacional con obras específicamente pianísticas compuestas para piano solo, y para piano y orquesta ${ }^{35}$. Dicho proceso innovador se activó y expandió en 1855-56, en una especie de 'segunda etapa del proceso' hasta ahora ignorada mediante las actuaciones de un jovencísimo pianista, Oscar de la Cinna, educado en la 'técnica pianística clásica', quien, además de la capital, recorrió también capitales de provincia más pequeñas, en las cuales dio a conocer una muestra de las composiciones pianísticas germánicas clásicas y románticas que habían asentado el gusto por el piano y su música en practicamente toda Europa y América.

\section{BIBLIOGRAFÍA}

Alemany Ferrer, V. (2007). "La construcción de pianos en Valencia hasta inicios del siglo xx". Anuario Musical, 62, pp. 335-364.

Alemany Ferrer, V. (2010). El piano en Valencia en los años del cambio al siglo XX (1879-1916). Barcelona: Consejo Superior de Investigaciones Científicas - Institución 'Milà i Fontanals' (Monumentos de la Música Española, 79).

Alemany Ferrer, V. (2011). "La estancia de Isaac Albéniz en Valencia en agosto de 1882". Anuario Musical, 66, pp. 260-261.

Alemany Ferrer, V. (2014). "La estancia de Franz Liszt en España y su influencia sobre los conciertos de piano españoles celebrados durante la segunda mitad del siglo XIX". En F. Morabito (ed.), En pèlerinage avec Lisżt»: Virtuosos, Repertoire and Performing Venues in the 19th-Century Europe, pp. 151-165. Turnhout: Brepols Publishers (Speculum Musicae series, vol. 24).

Alonso González, C. (2001). "Un espacio de sociabilidad musical en la España romántica: las sociedades instructo-recreativas". Cuadernos de música iberoamericana, 8-9, pp. 17-40.

Álvarez Cañibano, A. (1996). Los papeles españoles de Glinka, 1845-1847: 150 aniversario del viaje de Mihail Glinka a España. Madrid: Consejería de Educación y Cultura, Comunidad de

\footnotetext{
35 En la década de 1860 los conciertos ofrecidos por Genaro Perrelli (1862), la gaditana Eloísa d’Herbil, y, entre otros, una jovencísima Teresa Carreño de trece años en Madrid y Zaragoza entre diciembre de 1866 y enero de 1867, seguían mostrando clara preferencia por la lírica. Carreño interpretó únicamente una balada de F. Chopin sin determinar y la Sonata 'Quasi una fantasia' en Do sostenido menor Op. 27 n. 2 'Claro de Luna' de L. van Beethoven junto a variadas obras propias y fantasías operísticas de F. Liszt, S. Thalberg y L. M. Gottschalk -su maestro-.
} 


\section{ViCTORIA ALEMANY}

Madrid, Centro de Documentación Musical, Ministerio de Educación y Cultura, Junta de Andalucía, Consejería de Cultura, Centro de Documentación Musical de Andalucía.

Álvarez Cañibano, A. (1999). “El viaje de Glinka por España”. En A. Alvarez Cañibano; P.V. Gutiérrez Dorado y C. Marcos Patiño (eds.), Relaciones musicales entre España y Rusia, pp. 77-100. Madrid: Centro de Documentación de Música y Danza, Instituto Nacional de las Artes Escénicas y de la Música (INAEM), Ministerio de Educación y Cultura.

Bordas Ibáñez, C. (2000). Instrumentos Musicales en Colecciones Españolas [2 vols.]. Madrid: Centro de Documentación de Música y danza (INAEM).

Bordas Ibáñez, C. (2002). "Piano. IV. Siglos XIX y XX”. En E. Casares (dir.), Diccionario de la música española e hispanoamericana, vol. 8, pp. 758-763. Madrid: SGAE.

Bordas Ibáñez, C. (2004). La producción y el comercio de instrumentos musicales en Madrid ca. 1770ca. 1870 [tesis doctoral]. Valladolid: Universidad de Valladolid.

Castillo, C. (1866). “Teresa Carreño en el Real Conservatorio de música y declamación”. Revista de bellas artes, 10, p. 6 (78).

Clementi, M. (1811). Introducción al Arte de tocar el Piano-Forte en que se hallan los elementos de la Música, Ideas preliminares para la dirección de los dedos, Ejemplos, Preludios, muchas Lecciones con los dedos marcados y mucha variedad de Ayres nacionales de España y de otros paises adaptados para el Piano-Forte. Sexta Edición. Dedicada a la Nación Española por el Autor, Muzio Clementi. Londres: Clementi-Banger-Hyde-Collard \& Davis [Davison impr.].

Cortizo Rodríguez, M. E. y Sobrino Sánchez, R. (2001). “Asociacionismo musical en España”. Cuadernos de música iberoamericana, 8-9, pp. 11-16

Cuervo, L. (2012). “José Nonó (1776-1845), compositor que fundó el primer Conservatorio de Música en Madrid”. Anuario Musical, 67, pp. 133-152.

Espín y Guillén, J. (1842). "Estudios biográficos. Artistas célebres españoles. Don José Miró". La Iberia Musical. Periódico Filarmónico de Madrid. Diario de los Artistas de las Sociedades y de los Teatros, dirigido por una Sociedad de Profesores, 21, pp. 2-3.

Ezquerro Esteban, A. (1998). "Salamero Reymundo, Francisco. Ensayo Biográfico sobre Diego Cera, un grausino universal" [recensión]. Revista de Musicología, 21/2, pp. 727733. 


\section{LA CONTRIBUCIÓN DE OSCAR DE LA CINNA A LA INTRODUCCIÓN DEL REPERTORIO PIANíSTICO EUROPEO EN ESPAÑA}

Ezquerro Esteban, A. (2002). "Nuevos datos para el estudio de los músicos Nebra en Aragón”. Anuario Musical, 57, pp. 113-156.

Fukushima, M. (2008). "Fabricantes de pianos en la Barcelona de 1900”. Recerca Musicològica, 17-18, pp. 279-297.

García Mallo, M. C. (2005). "Peters y España: edición musical y relaciones comerciales entre 1868 y 1892”. Anuario Musical, 60, pp. 115-167.

García Tena, D. F. (ed.) (1856). "Ha llegado a Sevilla el célebre pianista húngaro Oscar de la Cinna". Diario de Córdoba de comercio, industria, administración, noticias y avisos (12.10.1856), p. 2.

Gil, F. de A. (1855). "Concierto del pianista Oscar de la Cinna”. Gaceta musical de Madrid 16 p. 1.

Gut, S. (1987). "Les influences espagnoles dans l'oeuvre musicale de Franz Liszt”, en E. Casares, I. Fernández de la Cuesta y J. López-Calo (eds.): España en la música de occidente. Actas del Congresso Internacional celebrado en Salamanca, 29 de octubre-5 de noviembre de 1985: "año europeo de la música" [2 vols.], vol. 2. Madrid: Instituto Nacional de las Artes Escénicas y de la Música, pp. 225-230.

Hazen, F. (1989). Haz̧en y el piano en España, 175 años. Madrid: Hazen.

Kirkpatrick, R. (1953). Domenico Scarlatti. Princeton (NJ): Princeton University Press [traducido por C. Janés y J. M. Martín Triana (1985). Madrid: Alianza (Alianza Música, 24)].

Luengo Gutiérrez, P. (2008). “Epistolario del organero José Antonio Morón (1780-1785)". Archivo Hispalense. Revista histórica, literaria y artística, 91, pp. 276-278.

Masarnau, S. de (1847). "Estado actual de la música en España. Articulo III”. El Renacimiento, 16, p. 126.

Milinowski, M. (1988). Teresa Carreño. Caracas: Monte Ávila Editores (Grandes Biografías) [1 ${ }^{\text {a }}$ ed. en castellano, prólogo de J. A. Calcaño y notas de W. Guido y M. Milanca, Caracas, Edime, 1953].

Moreno Calderón, J. M. (2005). "Franz Liszt en Córdoba". Musicalia - Revista del Conservatorio Superior de Música 'Rafael Oroz̧co' de Córdoba, 3.

Pajares Barón, M. (1987). "Franz Liszt en Sevilla y en Cádiz (diciembre, 1844 - enero, 1845)". Revista de Musicología, 10/3, pp. 887-918. 


\section{ViCTORIA ALEMANY}

Rojas Buendía, M. del M. (2014). "La libertad de conciencia y el derecho de asociación en la Constitución española de 1812". Revista de historiografía (RevHisto), 20, pp. 41-58.

Rosa Jiménez, J. L. de la (2014). José Miró y Anoria (1815-1878) y la pedagogía pianística andaluza de su tiempo (Tesis Doctoral). Granada: Universidad de Granada.

Salas Villar, G. (1997). "Santiago de Masarnau y la implantación del piano romántico en España”. Cuadernos de música iberoamericana, 4, pp. 197-222.

Salas Villar, G. (1999). "Introducción”, sec. "Perfil biográfico de Santiago Masarnau Fernández", en: Piano romántico español: Santiago Masarnau y Pedro Albéniz, [edición crítica G. Salas]. Madrid: Instituto Complutense de Ciencias Musicales, (Música hispana. Serie C, Antologías, 6).

Fecha de recepción: 26/06/2018

Fecha de aceptación: 30/07/2018 